\title{
Time to Next Treatment, Health Care Resource Utilization, and Costs Associated with Ibrutinib Use Among U.S. Veterans with Chronic Lymphocytic Leukemia/Small Lymphocytic Lymphoma: A Real-World Retrospective Analysis
}

\author{
Qing Huang, PhD; Sujana Borra, MPH; Jieni Li, MPH; Li Wang, MA, PhD, MBA; Sulena Shrestha, MPH; \\ Murali Sundaram, PhD; and Nora Janjan, MD, MPSA, MBA
}

\begin{abstract}
BACKGROUND: Chronic lymphocytic leukemia/small lymphocytic lymphoma (CLL/SLL) is the most common adult leukemia, accounting for $\approx 37 \%$ of all leukemias in the United States. Limited real-word evidence is available on the outcomes of ibrutinib use among previously untreated patients in the U.S. Veterans Health Administration (VHA) population diagnosed with CLL/SLL.

OBJECTIVES: To (a) evaluate time to next treatment (TTNT) among U.S. veterans with CLL/SLL who initiated ibrutinib versus chemoimmunotherapy (CIT) in first line (1L) and $1 \mathrm{~L}$ ibrutinib versus ibrutinib in later lines (2L+) and (b) compare health care resource utilization (HRU) and costs between the $1 \mathrm{~L}$ ibrutinib and CIT cohorts.

METHODS: Adults with CLL/SLL and claims for $1 \mathrm{~L}$ single-agent ibrutinib or CIT (index date $=$ first prescription claim date) were included from Veterans Health Administration Data (April 1, 2013-March 31, 2018). A subset of the CIT $1 \mathrm{~L}$ cohort with evidence of ibrutinib in $2 \mathrm{~L} / 3 \mathrm{~L}$ was defined as the ibrutinib 2L+ cohort. Kaplan-Meier curves and Cox proportional hazard models were used to evaluate TTNT, and generalized linear models were used to determine all-cause per patient per month (PPPM) HRU and costs during $1 \mathrm{~L}$ among propensity score-matched (PSM) cohorts.
\end{abstract}

RESULTS: After PSM, 614 patients were included in each of the $1 \mathrm{~L}$ ibrutinib and $1 \mathrm{~L} \mathrm{CIT} \mathrm{cohorts,} \mathrm{and} 149$ were included in each of the $1 \mathrm{~L}$ ibrutinib and $2 \mathrm{~L}+$ ibrutinib cohorts. The $1 \mathrm{~L}$ ibrutinib cohort had significantly longer TTNT compared with each of the $1 \mathrm{~L} \mathrm{CIT} \mathrm{and} 2 \mathrm{~L}+$ ibrutinib cohorts $(P<0.0001$ and $P=0.0001$, respectively) and was less likely to have a next line of treatment than the CIT $1 \mathrm{~L}$ cohort ( $\mathrm{HR}=0.52 ; 95 \% \mathrm{Cl}=0.42-0.65 ; P<0.0001$ ) and the $2 \mathrm{~L}+$ ibrutinib cohort $(\mathrm{HR}=0.39 ; 95 \% \mathrm{Cl}=0.22-0.69 ; P=0.0012)$. The $1 \mathrm{~L}$ ibrutinib cohort had significantly fewer inpatient visits (rate ratio $[\mathrm{RR}]=0.38 ; 95 \% \mathrm{Cl}=0.28-0.52 ; P \leq 0.05$ ) and outpatient visits PPPM (RR $=0.72 ; 95 \% \mathrm{Cl}=0.68-0.77 ; P \leq 0.5)$ compared with the CIT $1 \mathrm{~L}$ cohort. Additionally, the $1 \mathrm{~L}$ ibrutinib cohort had $\$ 7,308$ significantly lower monthly medical costs $(95 \% \mathrm{Cl}=-\$ 9,892$ to $-\$ 4,895 ; P \leq 0.05)$ versus the $1 \mathrm{~L} \mathrm{CIT}$ cohort, resulting in comparable monthly total health care cost (medical and pharmacy) between real-world $1 \mathrm{~L}$ patients treated by ibrutinib and CIT $(-\$ 2,160 ; 95 \% \mathrm{Cl}=-\$ 4,840-\$ 347 ; P>0.05)$.

CONCLUSIONS: These findings demonstrate that among U.S. veterans with CLL/SLL, $1 \mathrm{~L}$ ibrutinib use was associated with significantly longer TTNT versus that of $1 \mathrm{~L} \mathrm{CIT.} \mathrm{Similarly,} \mathrm{early} \mathrm{treatment} \mathrm{with} \mathrm{ibrutinib} \mathrm{was}$ associated with longer TTNT as compared to ibrutinib use in later lines of therapy. Moreover, $1 \mathrm{~L}$ ibrutinib was associated with lower HRU and medical costs compared with $1 \mathrm{~L} \mathrm{CIT,} \mathrm{completely} \mathrm{offsetting} \mathrm{the} \mathrm{higher} \mathrm{pharmacy}$ costs related to $1 \mathrm{~L}$ ibrutinib treatment.

J Manag Care Spec Pharm. 2020;26(10):1266-75

Copyright $\odot 2020$, Academy of Managed Care Pharmacy. All rights reserved.

\section{What is already known about this subject}

Chronic lymphocytic leukemia/small lymphocytic lymphoma (CLL/SLL) is the most common adult leukemia, accounting for $\approx 37 \%$ of all leukemias in the United States.

Targeted therapies, like ibrutinib, have changed the therapeutic paradigm for CLL achieving improved outcomes with acceptable toxicity.

\section{What this study adds}

IL ibrutinib treatment, compared with chemoimmunotherapy or later administration of ibrutinib, is associated with significantly longer time to new treatment among U.S. veterans.

Lower health care resource utilization and medical costs with $1 \mathrm{~L}$ ibrutinib offset higher pharmacy costs incurred by U.S. veterans.

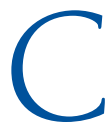
hronic lymphocytic leukemia (CLL) and small lymphocytic lymphoma (SLL) account for $\approx 7 \%$ of newly diagnosed non-Hodgkin lymphoma (NHL) cases. ${ }^{1}$ In 2019, the number of cases of newly diagnosed CLL in the United States was estimated to be 20,720, accounting for 1.2\% of all new cancer cases. ${ }^{2}$ Although some patients have a highly aggressive course, others have indolent disease that progresses slowly over a period of years and does not require immediate treatment. ${ }^{3,4}$ The most unfavorable prognosis is associated with the patients carrying deletion $17 \mathrm{p}$, deletion 11q, unmutated immunoglobulin heavy chain variable gene (IGHV), or mutation of the TP53 gene (mTP53). 5,6

The etiology of CLL/SLL is unknown. However, CLL incidence is 2 times higher in men, and nearly $70 \%$ of patients are aged $\geq 65$ years at diagnosis. ${ }^{7}$ Additionally, positive family history of CLL, European ancestry, and exposure to chemicals such as Agent Orange and pesticides are considered risk factors of CLL. ${ }^{8}$ Considering these risk factors, veterans have a higher risk for CLL as they are predominantly male, aged $\geq 65$ years, and have higher incidence of exposure to Agent Orange or other herbicides during military service. ${ }^{9}$ 


\section{Time to Next Treatment, Health Care Resource Utilization, and Costs Associated with Ibrutinib Use Among U.S. Veterans with Chronic Lymphocytic Leukemia/Small Lymphocytic Lymphoma: A Real-World Retrospective Analysis}

Chemoimmunotherapy (CIT) regimens-such as fludarabine, cyclophosphamide, and rituximab (FCR); bendamustine and rituximab (BR); and the combination of chlorambucil and cluster of differentiation (CD)20 antibodies-represented progress in the treatment of CLL with improved response and prolonged survival rates. ${ }^{10,11}$ Although CIT still plays a role in the treatment of previously untreated CLL patients, without TP53 mutations, dell7p and unmutated (U-)CLL cytogenetics, the treatment landscape has changed considerably with the introduction of oral targeted therapies..$^{10,12-14}$

In 2014, the U.S. Food and Drug Administration (FDA) approved ibrutinib, a once-a-day oral inhibitor of Bruton's tyrosine kinase, initially for the treatment of CLL after receiving $\geq 1$ previous therapies and subsequently as a first-line (1L) treatment for patients with del(17p). In 2016, the FDA expanded approval for ibrutinib as a $1 \mathrm{~L}$ treatment for all patients, based on the RESONATE-2 study. ${ }^{15,16}$ The results of additional clinical trials demonstrate that ibrutinib improves overall response, progression-free survival, and overall survival among treatment-naive CLL patients when compared with other commonly used chemoimmunotherapy regimens including FCR, BR, or the combination of chlorambucil and obinutuzumab. ${ }^{11,15,17-20}$ Until recently, ibrutinib was the only preferred regimen listed in National Comprehensive Cancer Network guidelines for 1L CLL treatment regardless of age, fitness, or comorbidities. ${ }^{16}$

Just as therapeutic approaches for CLL have evolved from CIT to targeted therapies, health care resource utilization and economic burden associated with CLL therapy will also evolve. The resource utilization and economic burden of CLL are significant, with monthly per-patient costs varying greatly by care setting, treatment regimen, and rate of adverse events (AEs). ${ }^{21-24}$ Compared with 2011, the number of people living with CLL in the United States is expected to increase by more than $50 \%$ by 2025 due to improved treatment options and higher survival rates. ${ }^{2,25}$

Given the limited real-world evidence of ibrutinib treatment among patients with CLL/SLL in the Veterans Administration (VA) population, the present study aimed to determine if treatment with ibrutinib single-agent in $1 \mathrm{~L}$ was beneficial in extending time to next treatment (TTNT) when compared with 1L CIT and ibrutinib in the second and third line (2L+) among veterans with CLL/SLL. Additionally, our study compared health care resource utilization (HRU) and costs between $1 \mathrm{~L}$ ibrutinib and 1L CIT cohorts.

\section{Methods}

\section{Study Design and Data Source}

This was a retrospective cohort study (Supplementary Figure 1, available in online article) utilizing pharmacy and medical claims from the VHA database during the period of April 1, 2013, through March 31, 2018. The VHA is the largest integrated health care system in United States, providing care at 1,250 health care facilities, including 172 VA medical centers and 1,069 outpatient sites of care of varying complexity to more than $\approx 9$ million veterans across the country. ${ }^{26}$

\section{Study Population}

Patients were required to have $\geq 2$ claims with a diagnosis code for CLL (International Classification of Diseases, Ninth Revision, Clinical Modification [ICD-9-CM]: 204.1; International Classification of Diseases, 10th Revision, Clinical Modification [ICD]10-CM: C91.1) or SLL (ICD-9-CM: 200.8; ICD-10-CM: C83.0) at any time during the study period (April 1, 2013-March 31, 2018). The first CLL/SLL diagnosis date was designated as the first observed CLL/SLL diagnosis date. Also, patients were required to have $\geq 1$ prescription claim for ibrutinib singleagent or any combination of National Comprehensive Cancer Network-recommended chemotherapy agents (bendamustine, chlorambucil, cyclophosphamide, cytarabine, doxorubicin, fludarabine, lenalidomide, oxaliplatin, pentostatin, vincristine, and cladribine) with an immunotherapy agent (alemtuzumab, obinutuzumab, ofatumumab, and rituximab) for CLL/SLL during the identification period (April 1, 2014-December 31, 2017) following the first observed CLL/SLL diagnosis date.

The identification period was chosen to capture the use of ibrutinib following U.S. approval. Ending the identification period 3 months before the end of data availability was intentional, to allow for $\geq 3$ months of follow-up data accumulation by the end-of-study period. The first prescription claim for ibrutinib single-agent or CIT at any time in the identification period following the first CLL/SLL diagnosis (initial diagnosis date) was designated as the index date. For combination regimens, agents prescribed within 30 days of the index date constituted 1L. Only the patients who were prescribed ibrutinib single-agent were considered, and those who received CD20 agents together with ibrutinib were excluded. Patients were also required to be aged $\geq 18$ years on the index date and have continuous health plan enrollment with medical and pharmacy benefits for $\geq 12$ months before and $\geq 30$ days after the index date. Patients were followed until death, end of continuous enrollment, or the end-of-study period, whichever occurred the earliest.

Patients were excluded from the study if they had evidence of ibrutinib or other CIT at any time before the index date and after the first diagnosis date or had evidence of pregnancy (ICD-9-CM: 630.xx-679.xx, v22.x-v24.x) or end-stage renal disease (ICD-9-CM: 585.6) at any time during the study period. Based on the $1 \mathrm{~L}$ treatment received patients were classified into the $1 \mathrm{~L}$ ibrutinib cohort or $1 \mathrm{~L}$ CIT cohort. In the $1 \mathrm{~L}$ CIT cohort, the subgroup of patients treated with a single-agent ibrutinib in the $2 \mathrm{~L} / 3 \mathrm{~L}$ and with continuous enrollment of $\geq 12$ months before and $\geq 30$ days after the index date was included in the $2 \mathrm{~L}+$ ibrutinib cohort (index date was defined as the date 


\section{Time to Next Treatment, Health Care Resource Utilization, and Costs Associated with Ibrutinib Use Among U.S. Veterans with Chronic Lymphocytic Leukemia/Small Lymphocytic Lymphoma: A Real-World Retrospective Analysis}

of initiating ibrutinib treatment, as presumed from the first claim for the drug).

\section{Demographic and Clinical Characteristics}

Demographic characteristics including age, sex, and race were measured on the index date and the year of the index treatment was reported. Quan-Charlson Comorbidity Index (Quan-CCI) score (Supplementary Table 1, available in online article), clinical conditions of interest (abdominal pain, anemia, autoimmune hemolytic anemia, enlarged lymph nodes, fatigue/ weakness, fever, lymphocytosis, thrombocytopenia, weight loss, atrial fibrillation, and major bleeding; diagnosis codes can be found in Supplementary Table 2, available in online article), and baseline medications related to common morbidities in the VHA population (antiplatelet drugs, anticoagulant agents, dexamethasone, prednisone, methylprednisolone, proton pump inhibitors, and antidepressants) were examined and reported in the 12-month pre-index period. Major bleeding was defined by the consensus report from the Bleeding Academic Research Consortium. ${ }^{27}$

\section{Outcome Measures}

TTNT was defined as the time from the index date to the initiation of next-line treatment. Patients lost to follow-up or without evidence of next-line treatment were censored. The $1 \mathrm{~L}$ therapy period spanned the index date through the earliest of addition or subtraction of any agent to the index regimen or initiation of a non-index regimen or resumption of the index regimen after a >90-day gap or death/end of continuous enrollment/study end. ${ }^{24}$ TTNT was compared between the $1 \mathrm{~L}$ ibrutinib and $1 \mathrm{~L}$ CIT cohorts as well as between $1 \mathrm{~L}$ ibrutinib and $2 \mathrm{~L}+$ ibrutinib cohorts.

All-cause HRU and health care costs per patient per month (PPPM) were measured during the $1 \mathrm{~L}$ therapy period for outpatient (including ER visits), inpatient, pharmacy services, and avoidable hospitalizations, as defined by the Agency for Healthcare Research and Quality (AHRQ) Prevention Quality Indicators (PQI; list of avoidable hospitalizations along with the diagnosis codes can be found in Supplementary Table 3 , available in online article). ${ }^{28}$ Among the causes for avoidable hospitalizations, defined by AHRQ PQI, are asthma, bacterial pneumonia, chronic obstructive pulmonary disease, dehydration, diabetes, heart failure, hypertension, perforated appendix, or urinary tract infection, outpatient visits resulting in hospitalization (outpatient visits followed by inpatient visits on the same day), and outpatient visits related to administration of antineoplastic agents. All-cause HRU and costs were compared between the 1L ibrutinib and 1L CIT cohorts only. As this study is a total cost of care analysis, all medical and pharmacy costs related to the disease and its treatment encountered by patients in each cohort were analyzed.

\section{Statistical Analysis}

Descriptive statistics were provided for all study variables (means and standard deviations [SDs] for continuous variables, numbers and percentages for categorical variables), among all study cohorts. Chi-square and Student t-tests were conducted to evaluate the statistical significance for categorical and continuous variables, respectively.

To reduce potential selection bias, 1-to-1 propensity score matching (PSM) was performed between the $1 \mathrm{~L}$ ibrutinib and $1 \mathrm{~L}$ CIT cohorts and between the $1 \mathrm{~L}$ ibrutinib and $2 \mathrm{~L}+$ ibrutinib cohorts, using nearest neighbor matching with a caliper width of 0.2 of the SD of the logit of the propensity score. Covariates used in propensity score calculation included demographics, index year, clinical conditions of interest (including those related to potential ibrutinib toxicity), and baseline medications. After PSM, the adequacy of the matching procedure was assessed by standardized difference (STD) of the baseline characteristics between the 2 groups. Characteristics with STD $<10 \%$ were considered well balanced. ${ }^{29}$

Kaplan-Meier (KM) curves and Cox proportional hazards models were used to evaluate the TTNT in the matched cohorts. Covariates were further adjusted in the models based on potential confounders in the literature along with covariates that were not balanced after PSM. Covariates adjusted in the Cox model for $1 \mathrm{~L}$ ibrutinib versus $1 \mathrm{~L}$ CIT included baseline inpatient length of stay (LOS) and all-cause total costs. Covariates adjusted in the Cox model for $1 \mathrm{~L}$ ibrutinib versus $2 \mathrm{~L}+$ ibrutinib included baseline demographic and clinical characteristics (age, race, index year, anemia, autoimmune hemolytic anemia, atrial fibrillation, dexamethasone), number of inpatient visits PPPM, number of outpatient visits PPPM, inpatient LOS, baseline allcause inpatient, outpatient and total costs.

Generalized linear models (GLM) were used to estimate the rate ratios (RR) and mean differences for HRU and costs PPPM, respectively, among the matched $1 \mathrm{~L}$ ibrutinib and $1 \mathrm{~L}$ CIT cohorts. Additionally, 95\% confidence intervals (CIs) were presented using the nonparametric bootstrap procedure with the percentile CI method. Similar to the Cox model for $1 \mathrm{~L}$ ibrutinib versus 1L CIT cohorts, baseline LOS and all-cause total costs were adjusted in the GLM.

\section{Sensitivity Analysis}

To ensure the robustness of the results in the main analysis, 3 sensitivity analyses were conducted. First, the PSM was repeated in all the study cohorts, adjusting for the index quarter of treatment (instead of index year), along with the demographics and clinical characteristics adjusted in the main analysis. Additionally, KM analysis, Cox modeling, and GLMs were repeated in these matched cohorts. Second, study cohort matching was performed using the inverse probability of treatment weighting (IPTW) approach, adjusting for the demographics, clinical characteristics, and index quarter. KM 


\section{Time to Next Treatment, Health Care Resource Utilization, and Costs Associated with Ibrutinib Use Among U.S. Veterans with Chronic Lymphocytic Leukemia/Small Lymphocytic Lymphoma: A Real-World Retrospective Analysis}

\begin{tabular}{|c|c|c|c|c|c|c|c|c|c|c|c|c|}
\hline \multirow[b]{2}{*}{ Baseline Characteristics } & \multicolumn{2}{|c|}{$\begin{array}{l}\text { Ibrutinib 1L } \\
\quad(n=614)\end{array}$} & \multicolumn{2}{|c|}{$\begin{array}{l}\text { CIT 1L } \\
(n=614)\end{array}$} & \multirow[b]{2}{*}{$P$ Value } & \multirow[b]{2}{*}{ STD, \% } & \multicolumn{2}{|c|}{$\begin{array}{l}\text { Ibrutinib 1L } \\
\quad(\mathbf{n}=149)\end{array}$} & \multicolumn{2}{|c|}{$\begin{array}{l}\text { Ibrutinib } 2 \mathrm{~L}+ \\
\quad(\mathrm{n}=149)\end{array}$} & \multirow[b]{2}{*}{$P$ Value } & \multirow[b]{2}{*}{ STD, \% } \\
\hline & Mean/n & $\mathrm{SD} / \%$ & Mean/n & $\mathrm{SD} / \%$ & & & Mean/n & $\mathrm{SD} / \%$ & Mean/n & $\mathrm{SD} / \%$ & & \\
\hline Age (in years) & 71.84 & 10.42 & 71.30 & 10.58 & 0.3684 & 5.14 & 73.89 & 12.18 & 70.50 & 9.94 & 0.0091 & 30.42 \\
\hline$<60$ & 38 & 6.19 & 45 & 7.33 & 0.4262 & 4.54 & NA & NA & 13 & 0.087248 & 0.2578 & 13.10 \\
\hline $60-64$ & 50 & 8.14 & 52 & 8.47 & 0.8362 & 1.18 & NA & NA & 14 & 0.09396 & 0.2778 & 12.56 \\
\hline $65-69$ & 197 & 32.08 & 201 & 32.74 & 0.8073 & 1.39 & 44 & 29.53 & 46 & 30.87 & 0.8008 & 2.91 \\
\hline $70-74$ & 159 & 25.90 & 151 & 24.59 & 0.5992 & 3.00 & 32 & 21.48 & 42 & 28.19 & 0.1800 & 15.53 \\
\hline $75-79$ & 82 & 13.36 & 79 & 12.87 & 0.7998 & 1.45 & 17 & 11.41 & 13 & 8.72 & 0.4412 & 8.90 \\
\hline$\geq 80$ & 88 & 14.33 & 86 & 14.01 & 0.8700 & 0.93 & 39 & 26.17 & 21 & 14.09 & 0.0093 & 30.37 \\
\hline \multicolumn{13}{|l|}{ Gender } \\
\hline Male & 610 & 99.35 & 607 & 98.86 & 0.3636 & 5.18 & 148 & 99.33 & 147 & 98.66 & 0.5617 & 6.70 \\
\hline \multicolumn{13}{|l|}{ Race } \\
\hline Caucasian & 488 & 79.48 & 482 & 78.50 & 0.6743 & 2.40 & 120 & 80.54 & 113 & 75.84 & 0.3261 & 11.36 \\
\hline African American & 82 & 13.36 & 90 & 14.66 & 0.5107 & 3.75 & 19 & 12.75 & 26 & 17.45 & 0.2574 & 13.10 \\
\hline Other or Unknown & 44 & 7.17 & 42 & 6.84 & 0.8230 & 1.28 & & & & A & & \\
\hline \multicolumn{13}{|l|}{ Index year } \\
\hline 2014 & 86 & 14.01 & 97 & 15.80 & 0.3781 & 5.03 & 17 & 0.114094 & $\mathrm{NA}$ & $\mathrm{NA}$ & 0.1578 & 16.37 \\
\hline 2015 & 130 & 21.17 & 131 & 21.34 & 0.9444 & 0.40 & 24 & 16.11 & 29 & 19.46 & 0.4488 & 8.75 \\
\hline 2016 & 194 & 31.60 & 195 & 31.76 & 0.9511 & 0.35 & 51 & 34.23 & 54 & 36.24 & 0.7160 & 4.20 \\
\hline 2017 & 204 & 33.22 & 191 & 31.11 & 0.4271 & 4.53 & 57 & 38.26 & 56 & 37.58 & 0.9050 & 1.38 \\
\hline $\begin{array}{l}\text { Quan Charlson } \\
\text { Comorbidity Index Score }\end{array}$ & 3.54 & 2.01 & 3.71 & 2.07 & 0.1466 & 8.29 & 3.80 & 2.24 & 3.80 & 2.19 & 0.9958 & 0.06 \\
\hline \multicolumn{13}{|c|}{ Clinical conditions of interest } \\
\hline Abdominal pain & 45 & 7.33 & 42 & 6.84 & 0.7386 & 1.90 & 13 & $8.72 \%$ & $\mathrm{NA}$ & $\mathrm{NA}$ & 0.6703 & 4.92 \\
\hline Anemia & 158 & 25.73 & 165 & 26.87 & 0.6500 & 2.59 & 40 & 26.85 & 56 & 37.58 & 0.0473 & 23.05 \\
\hline $\begin{array}{l}\text { Autoimmune hemolytic } \\
\text { anemia }\end{array}$ & 12 & 1.95 & 14 & 2.28 & 0.6918 & 2.26 & NA & NA & NA & NA & 0.3099 & 11.75 \\
\hline Enlarged lymph nodes & 112 & 18.24 & 105 & 17.10 & 0.6005 & 2.99 & 32 & 21.48 & 28 & 18.79 & 0.5634 & 6.68 \\
\hline Fatigue/weakness & 49 & 7.98 & 45 & 7.33 & 0.6677 & 2.45 & 22 & 14.77 & 20 & 13.42 & 0.7392 & 3.85 \\
\hline Fever & 12 & 1.95 & 16 & 2.61 & 0.4445 & 4.36 & $\mathrm{NA}$ & $\mathrm{NA}$ & $\mathrm{NA}$ & $\mathrm{NA}$ & 0.4537 & 8.66 \\
\hline Lymphocytosis & 19 & 3.09 & 19 & 3.09 & 1.0000 & 0.00 & $\mathrm{NA}$ & $\mathrm{NA}$ & $\mathrm{NA}$ & $\mathrm{NA}$ & 1.0000 & 0.00 \\
\hline Thrombocytopenia & 82 & 13.36 & 89 & 14.50 & 0.5640 & 3.29 & 25 & 16.78 & 26 & 17.45 & 0.8778 & 1.78 \\
\hline Weight loss & 40 & 6.51 & 43 & 7.00 & 0.7331 & 1.94 & 16 & 10.74 & 14 & 9.40 & 0.7002 & 4.45 \\
\hline Atrial fibrillation & 49 & 7.98 & 52 & 8.47 & 0.7553 & 1.78 & 17 & 11.41 & NA & $\mathrm{NA}$ & 0.1578 & 16.37 \\
\hline Major bleeding & 26 & 4.23 & 24 & 3.91 & 0.7727 & 1.65 & & & & A & & \\
\hline \multicolumn{13}{|l|}{ Baseline medications } \\
\hline Antiplatelet drugs & 141 & 22.96 & 140 & 22.80 & 0.9458 & 0.39 & 27 & 18.12 & 29 & 19.46 & 0.7668 & 3.42 \\
\hline Anticoagulant agents & 52 & 8.47 & 60 & 9.77 & 0.4278 & 4.52 & 18 & 12.08 & 19 & 12.75 & 0.8606 & 2.03 \\
\hline Dexamethasone & 20 & 3.26 & 26 & 4.23 & 0.3672 & 5.14 & 18 & 12.08 & 30 & 20.13 & 0.0586 & 21.97 \\
\hline Prednisone & 83 & 13.52 & 87 & 14.17 & 0.7410 & 1.88 & 34 & 22.82 & 36 & 24.16 & 0.7846 & 3.16 \\
\hline Methylprednisolone & 19 & 3.09 & 16 & 2.61 & 0.6069 & 2.93 & $\mathrm{NA}$ & $\mathrm{NA}$ & 12 & 8.05 & 0.6577 & 5.12 \\
\hline Proton pump inhibitors & 217 & 35.34 & 208 & 33.88 & 0.5893 & 3.08 & 61 & 40.94 & 59 & 39.60 & 0.8133 & 2.73 \\
\hline Antidepressants & 30 & 4.89 & 29 & 4.72 & 0.8939 & 0.76 & & & & A & & \\
\hline
\end{tabular}

analysis, Cox modeling, and GLMs were repeated in these matched cohorts.

\section{Results}

After applying the sample selection criteria, there were 787 patients in the $1 \mathrm{~L}$ ibrutinib cohort and 1,039 patients in the $1 \mathrm{~L}$
CIT cohort (Supplementary Table 4, available in online article) Of these 1L CIT patients, 597 patients initiated BR (57.5\%), 150 initiated FCR (14.4\%), and 94 initiated chlorambucilobinutuzumab (9.0\%). Additionally, among the $1 \mathrm{~L}$ ibrutinib patients, 146 (18.6\%) received 2L therapy, of which 92 (63.0\%) restarted ibrutinib as a $2 \mathrm{~L}$ regimen (after a $>90$-day gap). In 
Time to Next Treatment, Health Care Resource Utilization, and Costs Associated with Ibrutinib Use Among U.S. Veterans with Chronic Lymphocytic Leukemia/Small Lymphocytic Lymphoma: A Real-World Retrospective Analysis

\section{FIGURE 1 Comparison of TTNT Between 1L Ibrutinib and 1L CIT}

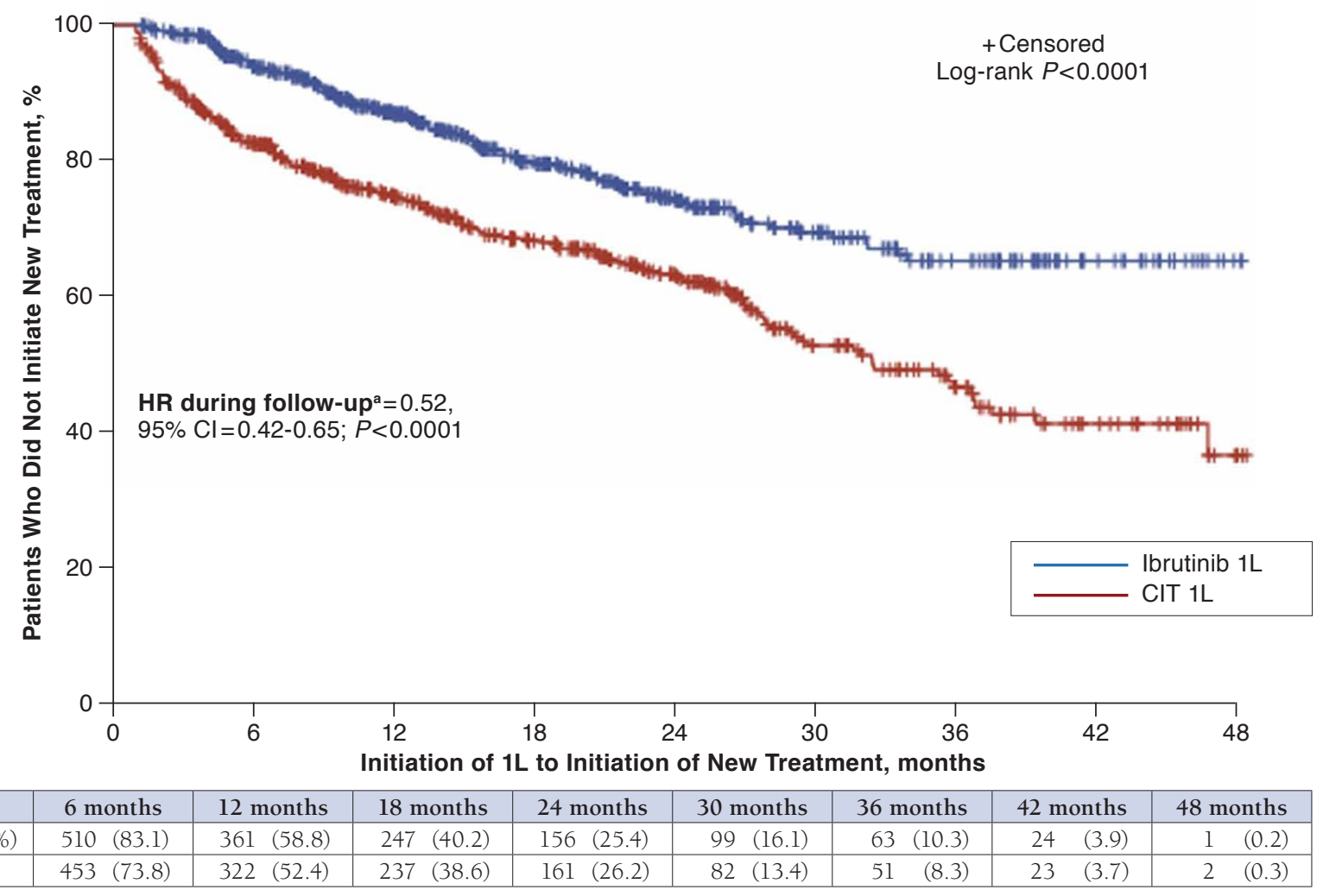

${ }^{a}$ Hazard ratios were calculated using Cox proportional hazards regression models in the propensity score-matched cohorts.

$1 \mathrm{~L}=$ first line; $2 \mathrm{~L}=$ second line; $C I=$ confidence interval; $H R=$ hazard ratio; $C I T=$ chemoimmunotherapy; $T T N T=$ time to next treatment.

contrast, among the 1L CIT patients, 409 (39.4\%) received a subsequent line of therapy, of which 185 (45.2\%) had ibrutinib as the $2 \mathrm{~L} / 3 \mathrm{~L}$ regimen.

\section{Baseline Characteristics Before PSM}

Compared with the CIT $1 \mathrm{~L}$ cohort, patients in the $1 \mathrm{~L}$ ibrutinib cohort were significantly older, and the majority were male and Caucasian. Additionally, the $1 \mathrm{~L}$ ibrutinib cohort had significantly lower mean Quan-CCI scores and a lower proportion of patients with anemia, enlarged lymph nodes, fatigue/ weakness, fever, and lymphocytosis, as well as a significantly lower proportion of patients with baseline medications including anticoagulant agents, dexamethasone, prednisone, and methylprednisolone versus the CIT 1L cohort (Supplementary Table 1, available in online article).

A similar trend was observed when demographic and clinical characteristics between the $1 \mathrm{~L}$ ibrutinib versus $2 \mathrm{~L}+$ ibrutinib cohorts were compared (Supplementary Table 1).

\section{Baseline Characteristics After PSM}

After PSM, a total of 614 patients each were included in the 1L ibrutinib and IL CIT cohorts and were well balanced with respect to all demographic and clinical characteristics examined (Table 1). Additionally, 149 patients were included in each of the $1 \mathrm{~L}$ ibrutinib versus $2 \mathrm{~L}+$ ibrutinib cohorts, the cohorts were well balanced for most baseline characteristics. The remaining differences, due to limited sample size, were adjusted during multivariate analysis of outcomes.

The mean length of follow-up period was 19.8 months (SD 12.0 months; median 17.6 months) in $1 \mathrm{~L}$ ibrutinib and 21.5 months (SD 12.8 months; median 20.2 months) in 1L CIT cohort. The duration of $1 \mathrm{~L}$ therapy period (active treatment period+no treatment period before starting 2L) was 17.4 months (SD 11.6 months; median 14.4 months) in 1L ibrutinib and 15.9 months (SD 12.1 months; median 13.0 months) in 1L CIT cohort. In the $1 \mathrm{~L}$ ibrutinib versus $2 \mathrm{~L}+$ ibrutinib cohorts, the mean length of follow-up period was 16.7 months (SD 11.5 months; median 13.1 months) and 16.9 months (SD 10.5 months; median 14.8 months), respectively; the duration of $1 \mathrm{~L}$ 
Time to Next Treatment, Health Care Resource Utilization, and Costs Associated with Ibrutinib Use Among U.S. Veterans with Chronic Lymphocytic Leukemia/Small Lymphocytic Lymphoma: A Real-World Retrospective Analysis

FIGURE 2 Comparison of TTNT Between $1 \mathrm{~L}$ Ibrutinib and 2L+ Ibrutinib

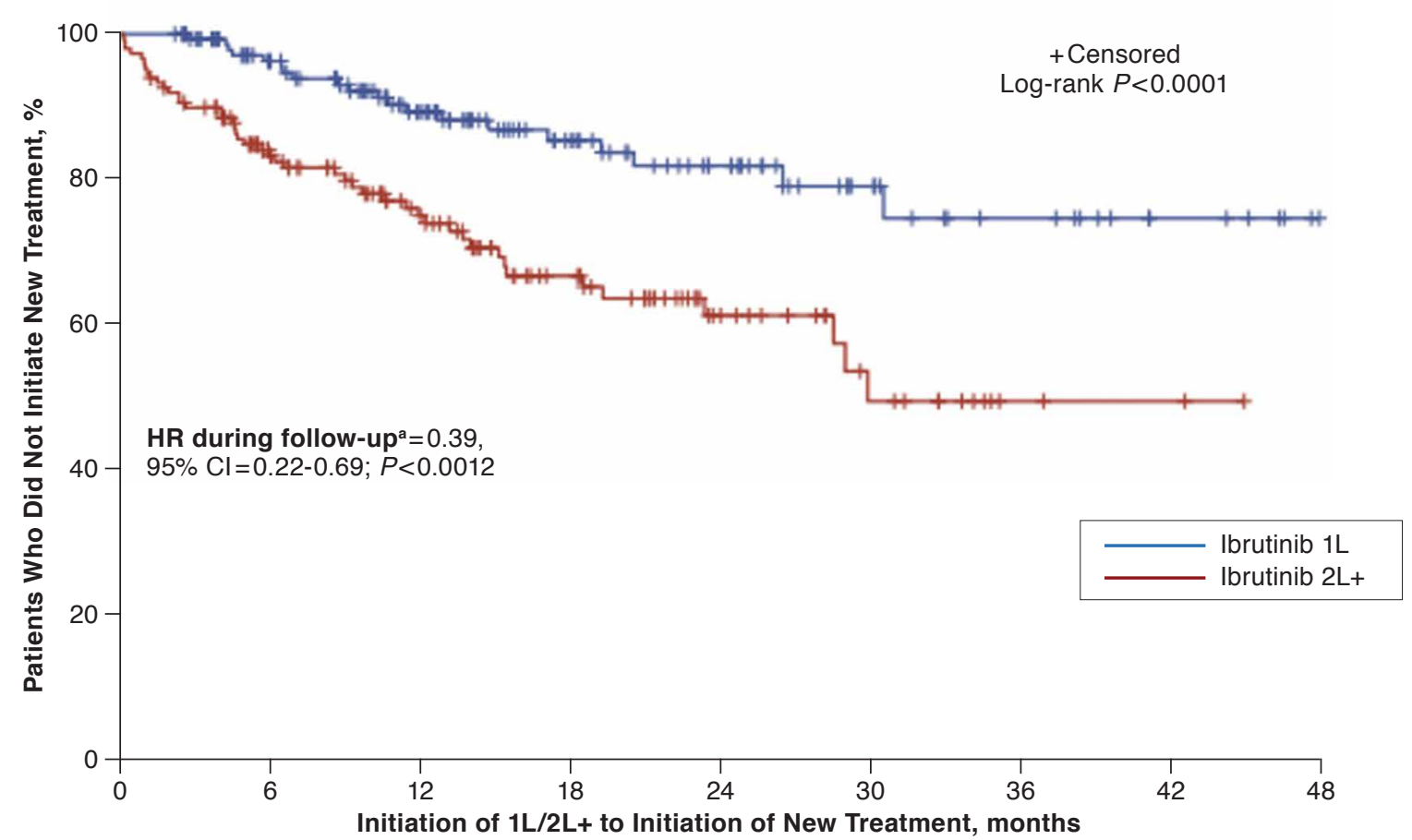

\begin{tabular}{|c|c|c|c|c|c|c|c|c|}
\hline Patie & 6 months & 12 months & 18 months & 24 months & 30 months & 36 months & 42 months & 48 months \\
\hline Ibrutinib lL, n (\%) & $122(81.9)$ & $86 \quad(57.7)$ & $56 \quad(37.6)$ & $38 \quad(25.5)$ & $21 \quad(14.1)$ & $13 \quad(8.7)$ & $6 \quad(4.0)$ & $(0)$ \\
\hline Ibrutinib 2L+, n (\%) & $104(69.8)$ & $73(49.0)$ & $46(30.9)$ & $24(16.1)$ & $(8.1)$ & $(2.0)$ & (1.3) & (0) \\
\hline
\end{tabular}

${ }^{a}$ Hazard ratios were calculated using Cox proportional hazards regression models in the propensity score-matched cohorts.

$1 \mathrm{~L}=$ first line; $2 \mathrm{~L}+=$ second line or later; $C I=$ confidence interval; $C I T=$ chemoimmunotherapy; HR=hazard ratio; $T T N T=$ time to next treatment.

therapy period was 15.9 months (SD 11.9 months; median 12.9 months) in the $1 \mathrm{~L}$ ibrutinib cohort and the duration on $2 \mathrm{~L} / 3 \mathrm{~L}$ therapy period was 13.7 months (SD 10.1 months; median 11.6 months) in the $2 \mathrm{~L}+$ ibrutinib cohort.

\section{TTNT in the Matched Cohorts}

KM analysis of TTNT revealed that $1 \mathrm{~L}$ ibrutinib patients had significantly longer TTNT than CIT $1 \mathrm{~L}$ or ibrutinib 2L+ patients (log-rank $P<0.0001$; Figure 1 and Figure 2). KaplanMeier estimates at landmark time points were presented in Table 1. Median TTNT was not reached in the $1 \mathrm{~L}$ ibrutinib cohort during the available follow-up. During the follow-up period, the hazard of initiating a new treatment was also lower for patients treated with $1 \mathrm{~L}$ ibrutinib versus $1 \mathrm{~L}$ CIT (48\% lower) or ibrutinib 2L+ (61\% lower; Figure 1 and Figure 2).

\section{GLM-Adjusted All-Cause HRU and Costs PPPM in the Matched Cohorts}

The 1L ibrutinib cohort had significantly lower all-cause HRU including inpatient ( $\mathrm{RR}=0.38,95 \% \mathrm{CI}=0.28-0.52 ; P \leq 0.05)$ and outpatient $(\mathrm{RR}=0.72,95 \% \mathrm{CI}=0.68-0.77 ; \mathrm{P} \leq 0.05)$ visits PPPM, and shorter LOS PPPM (RR $=0.46,95 \% C I=0.32-0.67$; $P \leq 0.05$ ) compared with the CIT 1 L cohort (Figure 3). Compared with the CIT $1 \mathrm{~L}$ cohort, the ibrutinib cohort had $\$ 7,308$ lower monthly all-cause medical costs $(95 \% \mathrm{CI}=-\$ 9,892$ to $-\$ 4,895 ; P \leq 0.05)$, mainly attributable to the lower monthly allcause outpatient costs $(-\$ 5,305 ; 95 \% \mathrm{CI}=-\$ 7,563$ to $-\$ 3,397$; $P \leq 0.05)$ followed by the monthly all-cause inpatient costs $(-\$ 1,243 ; 95 \% C I=-\$ 1,869$ to $-\$ 556 ; P \leq 0.05)$ (Figure 4). Monthly outpatient costs were driven mainly by the lower monthly costs related to the administration of antineoplastic agents $(-\$ 3,201 ; 95 \% C I=-\$ 6,781$ to $-\$ 1,984 ; P \leq 0.05)$. Higher all-cause pharmacy costs in the ibrutinib cohort $(\$ 5,204 ; 95 \%$ $\mathrm{CI}=\$ 4,875-\$ 5,489 ; \quad P \leq 0.05)$ were offset by lower medical costs, resulting in overall net monthly all-cause total cost savings of $\$ 2,160$ (95\% CI $=-\$ 4,840-\$ 347 ; P>0.05$ ); however, the difference was not statistically significant (Figure 4). 
Time to Next Treatment, Health Care Resource Utilization, and Costs Associated with Ibrutinib Use Among U.S. Veterans with Chronic Lymphocytic Leukemia/Small Lymphocytic Lymphoma: A Real-World Retrospective Analysis

FIGURE 3 GLM-Adjusted All-Cause HRU and Health Care Costs PPPM in 1L Ibrutinib Versus 1L CIT Cohorts

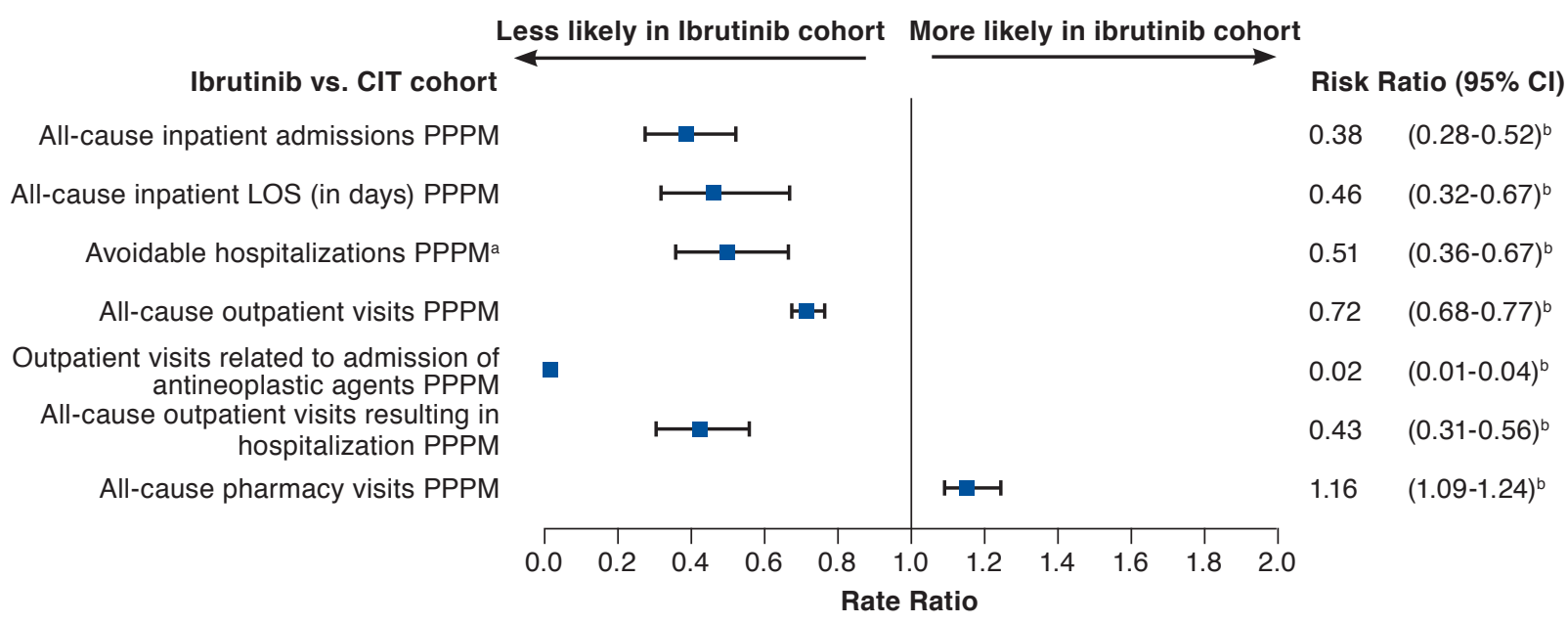

Note: Risk ratio was obtained from negative binomial model in the matched cohorts, adjusting for baseline LOS and total costs; $95 \%$ CI and P values were obtained from a nonparametric bootstrap procedure.

${ }^{a}$ Avoidable hospitalizations per AHRQ PQI included hospitalizations for asthma, bacterial pneumonia, chronic obstructive pulmonary disease, dehydration, diabetes, heart failure, hypertension, perforated appendix, or urinary tract infection.

bignificant at $P \leq 0.05$.

$1 L=$ first line; $A H R Q=$ Agency for Healthcare Research and Quality; $C I=$ confidence interval; $C I T=$ chemoimmunotherapy; GLM=generalized linear model; HRU =health care resource utilization; $L O S=$ length of stay; $P P P M=$ per patient per month; $P Q I=$ prevention quality indicators.

\section{Sensitivity Analysis}

Similar trends were observed with the PSM adjusted for the index quarter, as well as the IPTW approach, showing that $1 \mathrm{~L}$ ibrutinib had significantly longer TTNT compared with the CIT 1L (PSM $P<0.0001$; IPTW $P<0.0001$ ) and $2 \mathrm{~L}+$ ibrutinib cohorts (PSM $P=0.0015$; IPTW $P=0.0018$ ). Additionally, the $1 \mathrm{~L}$ ibrutinib cohort had significantly lower HRU and all-cause total medical costs but similar all-cause total costs compared with the CIT 1L cohort.

\section{Discussion}

This claims-based study reveals insights into the real-world treatment outcomes including TTNT, HRU, and costs among CLL/SLL patients in the VHA population. The results of this study show that $1 \mathrm{~L}$ ibrutinib is associated with a significantly longer TTNT than IL CIT and 2L+ ibrutinib. These data continue to support, in a real-world setting, the superior efficacy of ibrutinib over CIT treatments in $1 \mathrm{~L}$ as seen in ibrutinib clinical trials. ${ }^{11,18,20,30}$

The most critical determinants of therapeutic options are the presence of del(17p)/TP53 mutation, physical fitness/age, and duration of previous response with earlier treatment. ${ }^{31}$ In CLL, which is diagnosed predominantly in an elderly population, the toxicities of therapy are of special concern. Toxicities can result in the interruption of therapy, diminish the opportunity for response, and can result in morbidity.
These adverse patient outcomes can result in increased HCRU and cost. However, treatment with ibrutinib has been well tolerated, and the most common adverse events have generally been low grade and transient. ${ }^{25}$

Consistent with high rates of progression-free survival observed among ibrutinib patients in several clinical trials, ${ }^{11,15,17-20,32,33}$ it was observed that $73.7 \%$ and $65.4 \%$ of the patients who initiated ibrutinib single-agent in $1 \mathrm{~L}$ did not initiate a new treatment after 24 and 36 months of follow-up, respectively. It is acknowledged, however, that only $25 \%$ of our population was evaluated after 24 months and only $10 \%$ after 36 months of follow-up due to censoring events. For indolent disease such as CLL, longer TTNT may provide a meaningful endpoint for patients and is therefore an important outcome measure. ${ }^{34}$

Our findings align with the results of a previous real-world study that showed that ibrutinib had a longer TTNT versus CIT in $1 \mathrm{~L}$; however, the percentage of patients on ibrutinib in $1 \mathrm{~L}$ remaining on the same treatment was higher in the previous study $(\approx 89 \%)$ at 24 months of follow-up..$^{24}$ Possible explanations for the discrepancies could be difference in patient age, comorbidities, adherence, or study design. Nevertheless, both studies provided real-world evidence of the sustained progression-free survival benefits of ibrutinib over CIT in 1L. ${ }^{24,34}$

Notably, our study is unique in having a longer follow-up; we observed that at 48 months of follow-up, the percentage of 
Time to Next Treatment, Health Care Resource Utilization, and Costs Associated with Ibrutinib Use Among U.S. Veterans with Chronic Lymphocytic Leukemia/Small Lymphocytic Lymphoma: A Real-World Retrospective Analysis

FIGURE 4 GLM-Adjusted Health Care Costs PPPM in 1L Ibrutinib Versus 1L CIT Cohorts

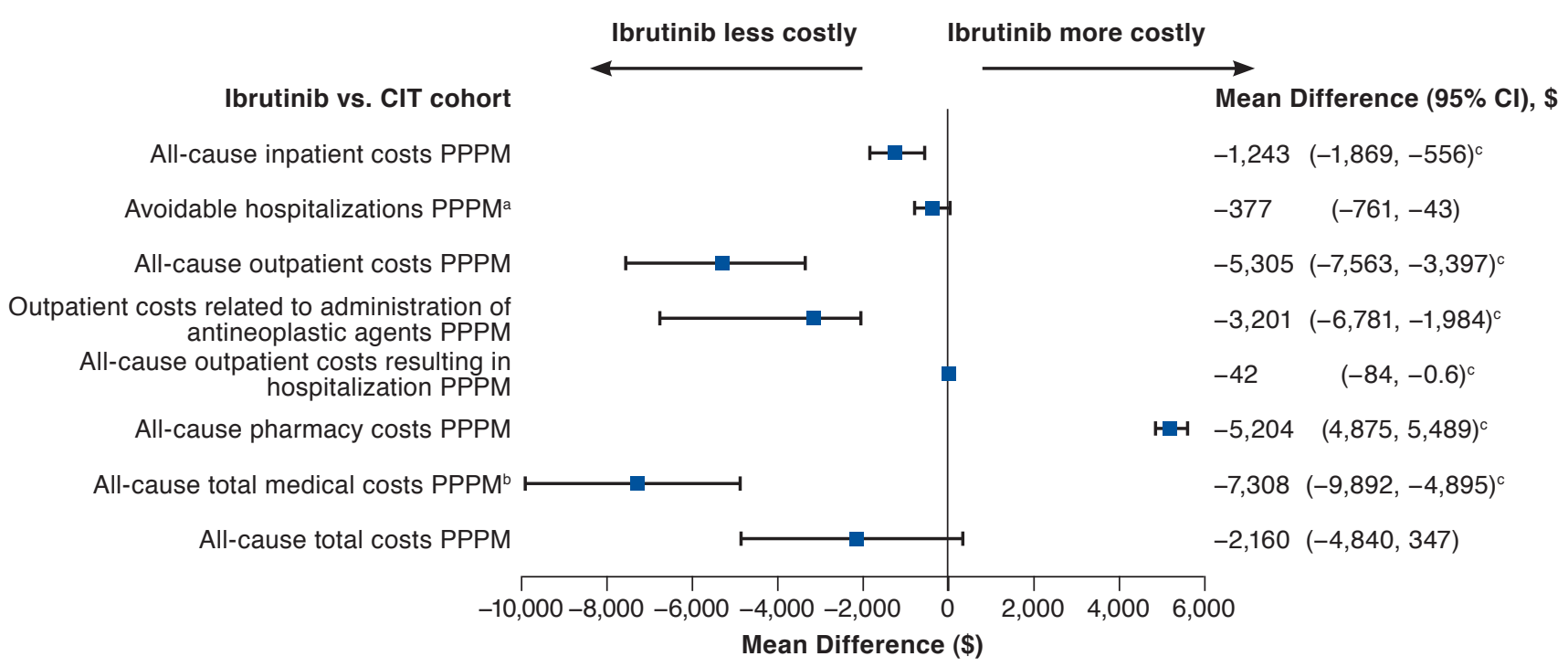

Note: Mean difference was calculated as the difference in the mean estimates obtained from GLM using gamma distribution and log link performed in the matched cohorts, adjusting for baseline LOS and total costs; 95\% CI and P values were obtained from a nonparametric bootstrap procedure.

${ }^{a}$ Avoidable hospitalizations per AHRQ PQI included hospitalizations for asthma, bacterial pneumonia, chronic obstructive pulmonary disease, dehydration, diabetes, heart failure, hypertension, perforated appendix, or urinary tract infection.

${ }^{b}$ All-cause total medical costs include inpatient and outpatient costs.

cSignificant at $P \leq 0.05$.

$1 \mathrm{~L}=$ first line; $A H R Q=$ Agency for Healthcare Research and Quality; $C I=$ confidence interval; $C I T=$ chemoimmunotherapy; GLM=generalized linear model; HRU=health care resource utilization; $L O S=$ length of stay; $P P P M=$ per patient per month; $P Q I=$ prevention quality indicators.

the 1 L ibrutinib cohort remaining free from needing a next-line treatment was only $8 \%$ lower than at 24 months, and, in the $1 \mathrm{~L}$ CIT cohort, twice the number of patients remained free from needing a next-line treatment at 48 months. We also found that patients who initiated ibrutinib in $1 \mathrm{~L}$ had significantly longer TTNT than patients with ibrutinib in 2L/3L, which suggests the potential benefit of early treatment with ibrutinib.

Consistent with the previous real-world study by Emond et al. (2019), ${ }^{24}$ the current study results show that $1 \mathrm{~L}$ ibrutinib was associated with lower all-cause HRU and medical costs compared with $1 \mathrm{~L}$ CIT. The total medical costs incurred by $1 \mathrm{~L}$ ibrutinib patients in the current study were approximately $68 \%$ lower than in the CIT $1 \mathrm{~L}$ cohort. Additionally, we observed that the estimates of total cost reduction in the ibrutinib cohort in IL support conclusions made by previous studies that found a significant difference in HRU and total costs between $1 \mathrm{~L}$ ibrutinib and $1 \mathrm{~L}$ CIT. ${ }^{24,36-39}$ Although the all-cause medical costs completely offset the pharmacy costs in the $1 \mathrm{~L}$ ibrutinib cohort, no statistical significance was observed in the all-cause total costs between $1 \mathrm{~L}$ ibrutinib and $1 \mathrm{~L}$ CIT cohorts.

\section{Limitations}

The findings from our study should be viewed in the context of some study limitations. The presence of a claim for a filled prescription does not indicate that the medication was consumed or taken as prescribed. The presence of a diagnosis code on a medical claim does not necessarily indicate a positive presence of disease, as the diagnosis code may be incorrectly coded or included as rule-out criteria rather indicating the actual presence of the disease; however, we sought to mitigate this by requiring $\geq 2$ diagnosis codes for CLL or SLL. Another limitation of claims data is that disease progression is not directly available. The most accepted approach used in cancer studies with claims data is to have TTNT act as a proxy for progression, although patients may start next treatment due to reasons other than progression, for example, intolerability. 6,7,25,27,38,40-42

Certain information that could influence study outcomes, such as clinical and disease-specific parameters, is not readily available in claims data. Also, there may be residual confounders due to unobserved or unrecorded clinical characteristics, as observed in any other claims-based study. It is recognized that the baseline clinical conditions are based on presence of specific diagnosis codes in the claims and the clinical 


\section{Time to Next Treatment, Health Care Resource Utilization, and Costs Associated with Ibrutinib Use Among U.S. Veterans with Chronic Lymphocytic Leukemia/Small Lymphocytic Lymphoma: A Real-World Retrospective Analysis}

presentations may be underreported. Ibrutinib was approved in 2014 for the 1L treatment of CLL/SLL patients; any 1L use before that time is either off-label or (likely) a misclassification of patients as new users due to the limited pre-index data available in the data source.

Additionally, it is important to note that del(17p)/TP53 mutation information for each patient is not available in VA claims. Also, the information of fluorescent in situ hybridization (FISH) testing was not extracted from the data, as evaluating the frequency of FISH testing was not a part of the study objectives. It has been shown that ibrutinib works well in treatment of CLL patients with $\operatorname{del}(17 \mathrm{p}) / \mathrm{TP} 53$ mutation whereas CIT does not. Therefore, the differences in outcomes between the subgroup of ibrutinib versus CIT patients with del(17p)/TP53 mutation would likely be even more prominent than the results presented in this study. ${ }^{11,41,42}$

Also, resource utilization information on HRU might not be complete if Medicare-eligible veterans use health care services outside the VA system. However, the VA provides more comprehensive prescription drug coverage than Medicare, so patients have a strong financial incentive to receive pharmacotherapy from VA providers.

Additionally, generalizability of the findings may be limited, as the current analysis was conducted with VHA data, which is a population with more comorbidities, with more limited access to care than the general population, and is predominantly composed of male patients with previous military service.

Finally, retrospective analysis of real-world use of therapies cannot eliminate selection bias. Physicians prescribe medications to patients who they think would benefit the most from the selected treatments, based on their clinical judgment, appetite for adopting innovation, and patient clinical characteristics. However, with proper adjustment of observable data elements and acknowledgement of potential limitations, realworld data can still provide meaningful insights from routine clinical practice.

\section{Conclusions}

The findings of our study show that newly diagnosed patients with CLL/SLL who initiated $1 \mathrm{~L}$ treatment with once-daily ibrutinib remained on treatment longer than those who initiated $1 \mathrm{~L}$ CIT or those using ibrutinib in later lines. Additionally, patients who initiated $1 \mathrm{~L}$ ibrutinib had lower HRU and medical costs as compared with those who initiated IL CIT. Further research with larger sample sizes and longer follow-up periods is needed to understand the evolving treatment patterns and outcomes of ibrutinib with its increased utilization in the $1 \mathrm{~L}$ for this predominantly older population with high unmet needs.

\section{Authors}

QING HUANG, PhD, and MURALI SUNDARAM, PhD, Janssen Scientific Affairs, Horsham, Pennsylvania. SUJANA BORRA, MPH; JIENI LI, MPH; LI WANG, MA, PhD, MBA; SULENA SHRESTHA, MPH; and NORA JANJAN, MD, MPSA, MBA, STATinMED Research, Plano, Texas.

AUTHOR CORRESPONDENCE: Qing Huang, PhD, MHS, Janssen Scientific Affairs, 800 Ridgeview Dr., Horsham, PA 19044 Tel.: 410.914.8216; Email: qhuang13@its.jnj.com.

\section{DISCLOSURES}

This research was sponsored by Janssen Scientific Affairs. The analyses were performed by STATinMED Research. Huang is an employee of Janssen Scientific Affairs and may own company stock. Sundaram was an employee of Janssen Scientific Affairs at the time this study was conducted. Borra and Janjan are employees of STATinMED Research, a paid consultant to the study sponsor. Wang, Li, and Shrestha were employees of STATinMED Research at the time this study was conducted.

\section{ACKNOWLEDGMENTS}

The authors thank Lorie Ellis of Janssen Scientific Affairs for her contribution to data analysis/interpretation and her insightful discussion. Editorial support was provided by Michael Moriarty of STATinMED Research.

\section{REFERENCES}

1. Armitage JO. A clinical evaluation of the International Lymphoma Study Group classification of non-Hodgkin's lymphoma. Blood. 1997:89(11):3909-18

2. National Institutes of Health. Cancer stat facts: leukemia-chronic lymphocytic leukemia. 2020. Available at: https://seer.cancer.gov/statfacts/ html/clyl.html. Accessed August 1, 2020.

3. Rai KR, Wasil T, Iqbal U, et al. Clinical staging and prognostic markers in chronic lymphocytic leukemia. Hematol Oncol Clin North Am. 2004;18(4):795-805.

4. Itchaki G, Brown JR. Experience with ibrutinib for first-line use in patients with chronic lymphocytic leukemia. Ther Adv Hematol. 2018;9(1):3-19.

5. Monic M, Stożek-Tutro A, Wladysiuk M, Hus I. Treatment options in chronic leukemia (CLL): a Polish perspective. J Health Pol Outcomes Res. 2018;1:20-33.

6. Strati P, Jain N, O'Brien S. Chronic lymphocytic leukemia: diagnosis and treatment. Mayo Clin Proc. 2018;93(5):651-64.

7. Gribben JG. How I treat CLL up front. Blood. 2010;115(2):187-97.

8. American Cancer Society. Chronic lymphocytic leukemia causes, risk factors, and prevention. May 10, 2018. Available at: https://www.cancer.org/ content/dam/CRC/PDF/Public/8680.00.pdf. Accessed August 1, 2020.

9. U.S. Department of Veterans Affairs. Public health, military exposures: chronic B-cell leukemias and Agent Orange. May 4, 2016. Available at: https://www.publichealth.va.gov/exposures/agentorange/conditions/bcellleukemia.asp. Accessed August 1, 2020

10. Chen Q, Jain N, Ayer T, et al. Economic burden of chronic lymphocytic leukemia in the era of oral targeted therapies in the United States. J Clin Oncol. 2017;35(2):166-74.

11. Woyach JA, Ruppert AS, Heerema NA, et al. Ibrutinib regimens versus chemoimmunotherapy in older patients with untreated CLL. N Engl J Med. 2018;379(26):2517-28. 


\section{Time to Next Treatment, Health Care Resource Utilization, and Costs Associated with Ibrutinib Use Among U.S. Veterans with Chronic Lymphocytic Leukemia/Small Lymphocytic Lymphoma: A Real-World Retrospective Analysis}

12. Rai KR, Jain P. Chronic lymphocytic leukemia (CLL): then and now. Am J Hematol. 2016;91(3):330-40.

13. Eichhorst B. Chronic lymphocytic leukemia: chemoimmunotherapy is over? No. Clin Lymphoma Myeloma Leuk. 2018;18(Suppl 1):S120-222.

14. Parikh SA. Chronic lymphocytic leukemia treatment algorithm 2018. Blood Cancer J. 2018;8(10):93.

15. Burger JA, Tedeschi A, Barr PM, et al. Ibrutinib as initial therapy for patients with chronic lymphocytic leukemia. New Engl J Med. 2015;373(25):2425-37.

16. National Comprehensive Cancer Network. Chronic Lymphocytic Leukemia/Small Lymphocytic Lymphoma V.1.2020. 2020. Available at: https://www.nccn.org/professionals/physician_gls/pdf/cll.pdf. Accessed August 4, 2020

17. Peterson D, Schwartz J. Ibrutinib in relapsed or refractory mantle cell lymphoma and chronic lymphocytic leukemia. J Adv Pract Oncol. 2014;5(5):348-54.

18. Robak T, Burger JA, Tedeschi A, et al. Single-agent ibrutinib versus chemoimmunotherapy regimens for treatment-naive patients with chronic lymphocytic leukemia: a cross-trial comparison of phase 3 studies. Am J Hematol. 2018;93(11):1402-10.

19. Byrd JC, Furman RR, Coutre SE, et al. Targeting BTK with ibrutinib in relapsed chronic lymphocytic leukemia. N Engl J Med. 2013;369(1):32-42.

20. Shanafelt TD, Wang XV, Kay NE, et al. Ibrutinib-rituximab or chemoimmunotherapy for chronic lymphocytic leukemia. N Engl J Med. 2019;381(5):432-43.

21. Kabadi SM, Goyal RK, Nagar SP, Kaye JA, Davis KL. Treatment patterns, adverse events, and economic burden in a privately insured population of patients with chronic lymphocytic leukemia in the United States. Cancer Med. 2019;8(8):3803-10

22. Chen Q, Jain N, Ayer T, et al. Economic burden of chronic lymphocytic leukemia in the era of oral targeted therapies in the United States.J Clin Oncol. 2017;35(2):166-74.

23. Jain N, Chen Q, Ayer T, et al. Prevalence and economic burden of chronic lymphocytic leukemia (CLL) in the era of oral targeted therapies. Clin Lymphoma Myeloma Leuk. 2015;15(Supp 2):S30.

24. Emond B, Sundaram M, Romdhani H, Lefebvre P, Wang S, Mato A. Comparison of time to next treatment, healthcare resource utilization, and costs in patients with chronic lymphocytic leukemia initiated on frontline ibrutinib or chemoimmunotherapy, clinical lymphoma, myeloma and leukemia. Clin Lymphoma Myeloma Leuk. 2019;19(12):763-75.e2

25. Stilgenbauer S, Furman RR, Zent CS. Management of chronic lymphocytic leukemia. Am Soc Clin Oncol Educ Book. 2015;35:164-75.

26. U.S. Department of Veterans Affairs. Veterans Health Administration: about VHA. Updated July 14, 2019. Available at: https://www.va.gov/health/ aboutvha.asp. Accessed August 4, 2020

27. Mehran R, Rao SV, Bhatt DL, et al. Standardized bleeding definitions for cardiovascular clinical trials: a consensus report from the Bleeding Academic Research Consortium. Circulation. 2011;123(23):2736-47.

28. Agency for Healthcare Research and Quality. Prevention quality indicators overview. 2020. Available at: http://www.qualityindicators.ahrq gov/Modules/pqi_resources.aspx. Accessed August 4, 2020.
29. Austin PC. Using the standardized difference to compare the prevalence of a binary variable between two groups in observational research. Commun Stat Simul Comput. 2009;38(6):1228-34.

30. Moreno C, Greil R, Demirkan F, et al. Ibrutinib plus obinutuzumab versus chlorambucil plus obinutuzumab in first-line treatment of chronic lymphocytic leukaemia (iLLUMINATE): a multicentre, randomised, openlabel, phase 3 trial. Lancet Oncol. 2019;20(1):43-56.

31. O'Brien SM, Jaglowski S, Byrd JC, et al. Prognostic factors for complete response to ibrutinib in patients with chronic lymphocytic leukemia: a pooled analysis of 2 clinical trials. JAMA Oncol. 2018;4(5):712-16.

32. Cuneo A, Follows G, Rigolin GM, et al. Efficacy of bendamustine and rituximab as first salvage treatment in chronic lymphocytic leukemia and indirect comparison with ibrutinib: A GIMEMA, ERIC and UK CLL FORUM study. Haematologica. 2018;103(7):1209-17.

33. Maddocks KJ, Ruppert AS, Lozanski G, et al. Etiology of ibrutinib therapy discontinuation and outcomes in patients with chronic lymphocytic leukemia. JAMA Oncol. 2015;1(1):80-87.

34. Roever L. Endpoints in clinical trials: advantages and limitations. Evidence Based Med Pract. 2016;2(1):1000el11.

35. Munir T, Brown JR, O'Brien S, et al. Final analysis from RESONATE: up to six years of follow-up on ibrutinib in patients with previously treated chronic lymphocytic leukemia or small lymphocytic lymphoma. Am J Hematol. 2019:94:1353-63

36. Dashputre AA, Gatwood KS, Gatwood J. Medication adherence, health care utilization, and costs among patients initiating oral oncolytics for multiple myeloma or chronic lymphocytic leukemia/small lymphocytic lymphoma. J Manag Care Spec Pharm. 2020;26(2):186-96. Available at: https://www.jmcp.org/doi/10.18553/jmcp.2020.26.2.186

37. Shanafelt TD, Borah BJ, Finnes HD, et al. Impact of ibrutinib and idelalisib on the pharmaceutical cost of treating chronic lymphocytic leukemia at the individual and societal levels. J Oncol Pract. 2015;11(3):252-58.

38. Nabhan C, Nero D, Lee CH, Kish JK, Mato AR. Cost-effectiveness comparison between ibrutinib, chemotherapy, and chemoimmunotherapy in front-line treatment of chronic lymphocytic leukemia (CLL). Blood. 2018;132(Suppl 1):4757.

39. Nabhan C, Chung J, Mato AR, Kish J, Nero D. Comparison of costs and health care resource utilization (HRU) in chronic lymphocytic leukemia (CLL) patients treated with front-line ibrutinib or chemoimmunotherapy. Blood. 2017;130(Suppl 1):2111.

40. Fischer K, Bahlo J, Fink AM, et al. Long-term remissions after FCR chemoimmunotherapy in previously untreated patients with CLL: updated results of the CLL8 trial. Blood. 2016;127(2):208-15.

41. Joffe E, Goldschmidt N, Bairey O, et al. Outcomes of second-line treatment after fludarabine cyclosphosphamide and rituximab in patients with chronic lymphocytic leukemia outside clinical trials. Eur J Hematol. 2018;101(3):399-406

42. Hillmen P, Rawstron AC, Brock K, et al. Ibrutinib plus venetoclax in relapsed/refractory chronic lymphocytic leukemia: the CLARITY Study. J Clin Oncol. 2019;37(30):2722-29. 